\title{
No Need of Fascia Closure to Reduce Trocar Site Hernia Rate in Laparoscopic Surgery: A Prospective Study of 200 Non-Obese Patients
}

\author{
Rikki Singal ${ }^{\mathrm{a}, \mathrm{d}}$, Muzzafar Zaman ${ }^{\mathrm{a}}$, Amit Mittal ${ }^{\mathrm{b}}$, Samita Singal ${ }^{\mathrm{b}}$, Karamjot Sandhu ${ }^{\mathrm{a}}$, \\ Anshu Mittal ${ }^{\mathrm{c}}$
}

\begin{abstract}
Background: Laparoscopy is widely practiced and offers realistic benefits over conventional surgery. Port closure is important after a laparoscopic procedure to prevent port site incisional hernia. Larger port size and increasing numbers of ports needed to perform more complex laparoscopic procedures are likely to increase the incidence of port site hernias (PSHs). PSHs tend to develop more frequently at umbilical and midline port sites due to the thinness of the umbilical skin and weaknesses in the linea alba. More than $90 \%$ of PSHs occur through $10 \mathrm{~mm}$ and large ports can occur through $5 \mathrm{~mm}$ ports also. The aim was to study the outcomes and complications in laparoscopic surgery without fascial sheath closure of port site. We compared the results with another group in which fascial closure was done by a standard method.
\end{abstract}

Methods: This was a prospective study carried out in the Department of Surgery, MMIMSR, Mullana, Ambala, from August 2013 to 2015 in a single unit by a single surgeon. A total of 200 patients were selected randomly for the different laparoscopic procedures. Patients were divided into group A (only skin closure was done without fascia closure) and group B (fascial closure of the port in addition to skin closure). In both groups, we used blunt trocar for the $10 \mathrm{~mm}$ port. Skin of the $5 \mathrm{~mm}$ port was closed simply. The results in two groups were compared in terms of complications like PSH, bleeding, and wound infection.

Results: The outcomes in two groups were compared with and without fascia closure of $10 \mathrm{~mm}$ trocar port site. Patients operated for lap cholecystectomy were $170(85 \%), 10(5 \%)$ for lap appendicectomy, and $20(10 \%)$ for lap hernia. The study compared the results in two

Manuscript accepted for publication August 26, 2016

aDepartment of Surgery, Maharishi Markandeshwer Institute of Medical Sciences and Research, Mullana, Distt-Ambala, Haryana, India

${ }^{b}$ Department of Radiodiagnosis and Imaging, Maharishi Markandeshwer Institute of Medical Sciences and Research, Mullana, Distt-Ambala, Haryana, India

'Department of SPM, Maharishi Markandeshwer Institute of Medical Sciences and Research, Mullana, Distt-Ambala, Haryana, India

${ }^{\mathrm{d} C}$ Corresponding Author: Rikki Singal, Dr Kundan Lal Hospital, Ahmedgarh, Distt-Sangrur, Punjab 148021, India. Email: singalsurgery@yahoo.com

doi: http://dx.doi.org/10.14740/gr715w groups mainly for PSH formation. The P value was insignificant and Fischer's exact test result came as 1.00 . There were no significant differences between the two groups in terms of PSH, bleeding and infection in non-obese cases.

Conclusion: In both groups, blunt trocar was introduced into the abdomen. We concluded that this is safe, without visceral injury, and no bleeding was seen in both the groups. We had not encountered any case with PSH formation in follow-up of $6-8$ months. There was no infection over the port site.

Keywords: Lap cholecystectomy; Port fascia closure; Laparoscopic visualization; Hernias

\section{Introduction}

Port site hernias (PSHs) are rare complications that occur in approximately $1 \%$ of all laparoscopic surgeries. With the use of bladeless, blunt-tipped entry trocars, some surgeons have argued that all port sites do not require fascial closure. Several cases of PSHs, however, have been reported recently with the use of bladeless trocars. They evaluated the incidence of PSH after robot-assisted radical prostatectomy with the fascial closure of only the midline 12-mm port site [1]. The development of non-bladed obturators with integrated stability sleeves allows for creation of a muscle-splitting dilated laparoscopic port site with minimal abdominal wall defects after removal of trocar sleeves [2]. It is recommended in literature that preventive measures should be taken to prevent PSHs after laparoscopic surgery [3]. Trocar site hernia (TSH) is defined as an incisional hernia which occurs after minimally invasive surgery on the trocar incision site [4].

Many techniques and devices have been introduced into practice to minimize the risk of port site complications, which occur in 1-6\% of cases [5]. The PSH depends on the trocar diameter, the trocar design, pre-existing fascial defects, and the direction of the port insertion. The risk of TSH is greater in obese and bariatric patients because of the larger preperitoneal space and elevated intraabdominal pressure and some authors advise closure of holes $>5 \mathrm{~mm}$ at the fascial level. The various methods for port closure after laparoscopic surgery are: 1) standard closure (via skin wound); 2) laparoscopic direct 


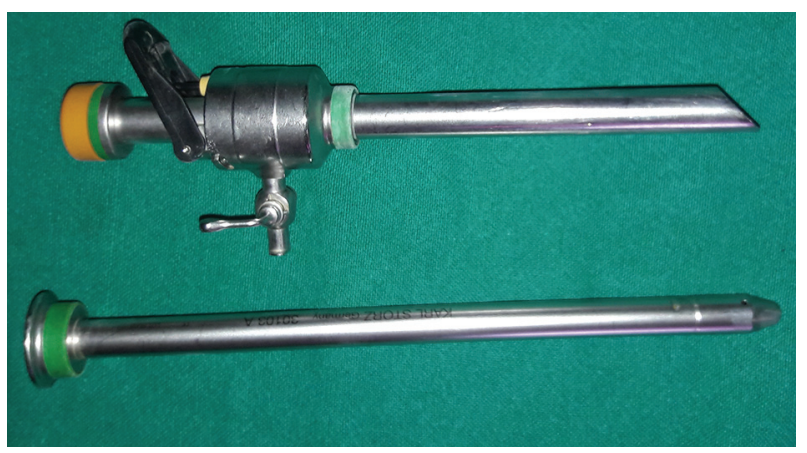

Figure 1. Blunt trocar (BT) was used to enter the abdomen after creation of the pneumoperitoneum.

visualization fascial closure methods; 3 ) using a spring-loaded needle or suture passer needle; and 4) angiocatheter technique. Port site infection is a type of surgical site infection that can occur at trocar site in laparoscopic surgery. Complications related to port sites are most commonly reported to be incisional hernias [6-8].

\section{Materials and Methods}

This is a prospective study of 200 patients who underwent different laparoscopic procedures in Department of Surgery in a single unit by a single surgeon from July 2013 to August 2015. Patients aged $>12$ and $<70$ years, without major systemic illnesses, and non-obese were included. Patients with past history of laparotomy, pregnancy, umbilical granuloma or hernia, obese, with recurrent incisional hernia, on steroids, and those unfit for laparoscopic surgery were excluded. A less than $1 \mathrm{~cm}$ infraumblical or supraumbilical skin incision was given according to surgery and blunt trocar (BT) (nonsharped trocar) was used to enter the abdomen for camera port. Other three ports were made of $5 \mathrm{~mm}$. In group A, fascia was not closed and only skin was closed. In group B, fascial sheath was closed with absorbable vicryl 1-0 and skin was closed. In both the groups, we did not use any other instrument to open the sheath (e.g. artery forcep to separate the fat or sheath); without any handling to the sheath fibers, abdomen was entered. The results in two groups were compared using Fischer's exact test.

\section{Results}

A total of 200 patients were included, out of whom 170 underwent laparoscopic cholecystectomy, 20 underwent laparoscopic hernia repair and 10 underwent laparoscopic appendectomy. The patients were divided into two groups A and B. In all the cases, BT was used to enter the abdomen after creation of the pneumoperitoneum (Fig. 1). In group A, upon completing the procedure, fascial sheath was not closed; while as in group B, sheath closure was done using standard method. In both groups, the direction for insertion of the $\mathrm{BT}$ was first in $90^{\circ}$ directions then while piercing the sheath, we changed the direction of the
Table 1. Complications in Two Groups

\begin{tabular}{llllll}
\hline & Complications & Group A & Group B & Test & P-value \\
\hline 1 & Port site hernia & 0 & 0 & 1.00 & $>0.05$ \\
2 & Bleeding & 0 & 0 & 1.00 & $>0.05$ \\
3 & Infection & 1 & 2 & 1.00 & $>0.05$ \\
\hline
\end{tabular}

trocar towards the pelvis so that it should not straight into the abdomen. Another thing we observed is that with the BT, you do not cut the sheath fibers just separate the fibers. But in case of sharp trocar, there will be tearing (cut) of the fibers due to which strength of the sheath fibers lost and blood supply also decreased which led to hernia formation. Only skin was closed in other three $5 \mathrm{~mm}$ ports. Apart from minor complaints, not much difference was observed immediately in terms of complications like PSH, bleeding, and visceral injury in these two groups. There was infection seen only in two cases in group B that was insignificant. As per the Fischer's exact test, the value came as 1.00 and $\mathrm{P}$ value was non-significant $(>0.5)$. After the surgery, every patient was followed up within $3-6$ months. We observed that we have not any major complications in both the groups like infection or hernia formation (Table 1).

\section{Discussion}

Minimal invasive surgery or key hole surgery has become the method of preference for the treatment of uncomplicated gall bladder stones, hernia repair and appendectomies. One of the key steps in the whole procedure is creation of pneumoperitoneum, which has gotten adverse hemodynamic and physiological effects [9]. After laparoscopic surgery, there are chances of TSH formation but data are sparse and based mostly on retrospective studies with a short and poorly defined follow-up period. TSH is commonly seen $(96 \%)$ in trocar sites of a minimum of $10 \mathrm{~mm}$, located often in the umbilicus region (82\%) [10]. The excitement to develop new techniques, to improve cosmesis and hasten recovery, has given rise to the natural orifice transluminal endoscopic surgery (NOTES), and more recently to single incision laparoscopic surgery (SILS). Furthermore, a single incision may decrease postoperative hernia rate [11]. It is seen that port site incisional hernia commonly occurred due to diameter of the cannula. In SILS technique, the fascial defect is larger than that in conventional laparoscopy and, in addition, multiple defects are converted to a single larger fascial defect, which increases PSH. Although it is unclear whether this technical maneuver translates into actual $\mathrm{PSH}$, there are several reports in the literature that suggest that prolonged manipulation coupled with reinsertion of the port may be associated with an enhanced risk of PSH [12].

Newer ports and bladeless trocars have decreased the incidence of TSH to nearly $0 \%$. These new trocars are atraumatic and split, rather than cut, muscle fibers upon entry. After removal of port, the abdominal layers contract, resulting in a defect much smaller than the trocar diameter [13]. In our study, we have used BT and came out with good results as there was no occurrence of PSH. We have made skin incision shorter 
through which we comfortably inserted the BT. Our theory is if you used sharp edge trocar of $10 \mathrm{~mm}$, it will cut the fascial fibers which makes it necessary to close the defect to prevent PSH. But in BT, fascial fibers will just get separated without any cutting of the fibers. In BT, we keep the position of the trocar just perpendicular while entering into the peritoneum and after half enter, we make the BT position towards the pelvis. Even we do not handle the sheath with any instrument, e.g. with artery forcep to create the space. Though our results are different from other studies but as per our search in literature no one has mentioned about the use of BT except in few studies. Nowadays, laparoscopic surgery/minimally invasive surgery is the best choice and it causes less postoperative pain and early recovery because of minimal trauma to the patients during surgery. Two ports can be used as an alternative to the three-port laparoscopic procedure with acute appendicitis and favorable outcomes [14].

The choice of port closure after laparoscopy will depend on the individual patient characteristics (such as body habitus, number and size of ports), the surgeon's preference, and available resources. This choice should be individualized. For example, all ports $>10 \mathrm{~mm}$ should be closed. Some $5-\mathrm{mm}$ ports should be closed when the peritoneal and/or fascial defect has enlarged significantly. Enlarging the incision to allow proper closure should take precedence over obtaining a good cosmetic result. Port closure should include fascia and peritoneum and it is advised to check the abdominal side of each wound during fascial closure via the laparoscope $[15,16]$.

Our study shows $10 \mathrm{~mm}$ port created by BT without closure of the fascial sheath is not at risk for PSH, bleeding, or infection, thus suggesting using skin suturing only technique to close the ports without much manipulation in non-obese patients who under their first time laparoscopic surgery is sufficient. Our results are consistent with several previously studies. One more study used non-bladed laparoscopic trocars and concluded that it is safe with the ability to visualize dissection through the abdominal wall layers to create the smallest port dissection without bleeding or cutting muscle fibers. Taken together, the existing body of evidence indicates that the ability to split the abdominal wall musculature by trocar allows the surgeon to forego closure of the small fascial defect [2]. Another study documented that, access to the peritoneum via a bladeless, $12-\mathrm{mm}$ visual entry trocar had no intraoperative bowel or vascular injuries, no mortality, and an extremely low rate of TSH $(0.2 \%)$. In addition, the study reported that Endopath trocar system, reduces TSHs, decreases bowel obstruction, and eliminates the need for time-consuming fascial closure [17].

In conclusion, every attempt should be made to ensure the proper closure of port sites. Otherwise, the morbidity associated with a PSH will negate the expected benefits of the intended minimally invasive surgery. Our data suggest that non-obese patients undergoing their first laparoscopic surgery with 10 $\mathrm{mm}$ port created by trocar may not need fascia closure.

\section{Financial Disclosures}

None.

\section{Conflicts of Interest}

None.

\section{Funding Source}

None.

\section{References}

1. Kang DI, Woo SH, Lee DH, Kim IY. Incidence of portsite hernias after robot-assisted radical prostatectomy with the fascial closure of only the midline $12-\mathrm{mm}$ port site. J Endourol. 2012;26(7):848-851.

2. Liu CD, McFadden DW. Laparoscopic port sites do not require fascial closure when nonbladed trocars are used. Am Surg. 2000;66(9):853-854.

3. Owens M, Barry M, Janjua AZ, Winter DC. A systematic review of laparoscopic port site hernias in gastrointestinal surgery. Surgeon. 2011;9(4):218-224.

4. Pamela D, Roberto C, Francesco LM, Umberto M, Carla M, Vincenzo N, Stefano T, et al. Trocar site hernia after laparoscopic colectomy: a case report and literature review. ISRN Surg. 2011;2011:725601.

5. Khan A, Siddiqui MN, Ahmed M. A simple technique of umbilical port closure in laparoscopic cholecystectomy. Ann R Coll Surg Engl. 1993;75(6):440.

6. Shah PR, Naguib N, Thippeswammy K, Masoud AG. Port site closure after laparoscopic surgery. J Minim Access Surg. 2010;6(1):22-23.

7. Hussain A, Mahmood H, Singhal T, Balakrishnan S, Nicholls J, El-Hasani S. Long-term study of port-site incisional hernia after laparoscopic procedures. JSLS. 2009;13(3):346-349.

8. Botea F, Torzilli G, Sarbu V. A simple, effective technique for port-site closure after laparoscopy. JSLS. 2011;15(1):77-80.

9. Singal R, Singal RP, Sandhu K, Singh B, Bhatia G, Khatri A, Sharma BP. Evaluation and comparison of postoperative levels of serum bilirubin, serum transaminases and alkaline phosphatase in laparoscopic cholecystectomy versus open cholecystectomy. J Gastrointest Oncol. 2015;6(5):479-486.

10. Shaher Z. Port closure techniques. Surg Endosc. 2007;21(8):1264-1274.

11. Leblanc F, Champagne BJ, Augestad KM, Stein SL, Marderstein E, Reynolds HL, Delaney CP. Single incision laparoscopic colectomy: technical aspects, feasibility, and expected benefits. Diagn Ther Endosc. 2010;2010:913216.

12. Agaba EA, Rainville H, Ikedilo O, Vemulapali P. Incidence of port-site incisional hernia after single-incision laparoscopic surgery. JSLS. 2014;18(2):204-210.

13. Lee J, Zheng XJ, Ng CY. Trocar site hernias from using bladeless trocars: should fascial closure be performed? J Surg Case Rep. 2014;2014(5). 
14. Gupta R, Singal R, Sharda VK, Singh B, Ahluwalia JS, Bhatia G, Arora G. Two port laparoscopic assisted appendicectomy versus three port laparoscopic appendicectomy: A prospective study of 50 cases. Trop J Med Res. 2015;18:14-19.

15. Helgstrand F, Rosenberg J, Bisgaard T. Trocar site hernia after laparoscopic surgery: a qualitative systematic review. Hernia. 2011;15(2):113-121.

16. Ng WT. A full review of port-closure techniques. Surg Endosc. 2007;21(10):1895-1897.

17. Rosenthal RJ, Szomstein S, Kennedy CI, Zundel N. Direct visual insertion of primary trocar and avoidance of fascial closure with laparoscopic Roux-en-Y gastric bypass. Surg Endosc. 2007;21(1):124-128. 